\title{
On Computing Periodic Coprime Factorizations
}

\author{
A. Varga
}

\begin{abstract}
Numerically reliable state space algorithms are proposed for computing coprime factorizations of periodic descriptor systems. Two factorizations are considered for causal periodic descriptor systems: stable coprime factorizations and coprime factorizations with inner denominators. The corresponding algorithms are based on a recursive generalized periodic Schur method for eigenvalue assignment. For general descriptor periodic systems an algorithm is proposed to compute factorizations with causal (proper) factors. This algorithm relies on computing suitable right annihilators of appropriate extended systems. The proposed algorithms are lifting-free and generally applicable regardless the original periodic descriptor state space representations are minimal or not.
\end{abstract}

\section{PRELIMINARIES}

We consider linear periodic time-varying discrete-time descriptor systems of the form

$$
\begin{aligned}
E_{k} x(k+1) & =A_{k} x(k)+B_{k} u(k) \\
y(k) & =C_{k} x(k)+D_{k} u(k)
\end{aligned}
$$

where the matrices $E_{k} \in \mathbb{R}^{\nu_{k} \times n_{k+1}}, A_{k} \in \mathbb{R}^{\nu_{k} \times n_{k}}, B_{k} \in$ $\mathbb{R}^{\nu_{k} \times m}, C_{k} \in \mathbb{R}^{p \times n_{k}}, D_{k} \in \mathbb{R}^{p \times m_{k}}$ are periodic with period $N \geq 1$. The periodic system (1) will be alternatively denoted by the periodic quintuple $\mathcal{S}:=\left(E_{k}, A_{k}, B_{k}, C_{k}, D_{k}\right)$. The vector of state-dimensions $\mathbf{n}=\left[n_{1}, n_{2}, \cdots, n_{N}\right]$ characterizes the state-space order of the periodic system. We assume that $\sum_{k=1}^{N} \nu_{k}=\sum_{k=1}^{N} n_{k}$ is fulfilled. Periodic systems of this general forms have been considered in several works (see for example [1], [2], [3], [4]). In what follows we summarize some notations and definitions for periodic systems used throughout the paper.

\section{A. Causal periodic systems}

The case of causal systems, when $\nu_{k}=n_{k+1}$ and $E_{k}$ are invertible matrices, plays an important role in most of applications. For a causal system (1), we denote the monodromy matrix at time $k$ by

$$
\Psi_{E_{k}^{-1} A_{k}}:=E_{k+N-1}^{-1} A_{k+N-1} E_{k+N-2}^{-1} A_{k+N-2} \cdots E_{k}^{-1} A_{k} .
$$

The eigenvalues of $\Psi_{E_{k}^{-1} A_{k}}$ are called characteristic multipliers of the periodic pair $\left(E_{k}, A_{k}\right)$. Let $\mathbb{D}^{-}$and $\mathbb{D}^{+}$be two open subsets of the complex plane $\mathbb{C}$ representing the interior and the exterior of the unit disk centered in the origin, respectively. We say that $\mathcal{S}$ is stable (or equivalently the periodic pair $\left(E_{k}, A_{k}\right)$ is stable $)$ if all characteristic multipliers have moduli less then one (i.e., $\Lambda\left(\Psi_{E_{k}^{-1} A_{k}}\right) \subset$ $\left.\mathbb{D}^{-}\right)$. Note that $\Psi_{E_{k}^{-1} A_{k}}$ has always $n_{k}-\underline{n}$ null eigenvalues,

A. Varga is with the German Aerospace Center, DLR - Oberpfaffenhofen, Institute of Robotics and Mechatronics, D-82234 Wessling, Germany. Email: Andras.Varga@dlr.de where $\underline{n}:=\min \left\{n_{k}\right\}$. The rest of $\underline{n}$ eigenvalues of $\Psi_{E_{k}^{-1} A_{k}}$ form the core characteristic multipliers and are the same for all values of $k$. For non-causal systems (e.g., with $E_{k}$ singular or even non-square), similar notions like finite and infinite characteristic multipliers can be defined using liftingbased representations (see for example [2]).

The definitions used for reachability and observability of causal periodic descriptor systems are those of [1], which also apply for the more general non-causal case. For causal systems, we will use the following simpler definitions of stabilizability and detectability. The causal periodic system $\mathcal{S}$ is stabilizable if there exists a periodic $F_{k}$ of appropriate dimensions such that the periodic pair $\left(E_{k}, A_{k}+B_{k} F_{k}\right)$ is stable. Similarly, the causal periodic system $\mathcal{S}$ is detectable if there exists a periodic $L_{k}$ of appropriate dimensions such that the periodic pair $\left(E_{k}, A_{k}+L_{k} C_{k}\right)$ is stable.

\section{B. Similarity transformations}

Two periodic systems $\mathcal{S}:=\left(E_{k}, A_{k}, B_{k}, C_{k}, D_{k}\right)$ and $\widetilde{\mathcal{S}}:=\left(\widetilde{E}_{k}, \widetilde{A}_{k}, \widetilde{B}_{k}, \widetilde{C}_{k}, D_{k}\right)$ are called similar if the matrices of their state-space representations are related by

$\widetilde{E}_{k}=U_{k} E_{k} V_{k+1}, \widetilde{A}_{k}=U_{k} A_{k} V_{k}, \widetilde{B}_{k}=U_{k} B, \widetilde{C}_{k}=C_{k} V_{k}$,

with $U_{k}$ and $V_{k} N$-periodic nonsingular matrices. Two similar systems have the same input-output mapping.

\section{System couplings}

The series coupling of two periodic systems $\mathcal{S}_{1}=$ $\left(E_{k}^{(1)}, A_{k}^{(1)}, B_{k}^{(1)}, C_{k}^{(1)}, D_{k}^{(1)}\right)$ of order $\mathbf{n}_{1}$ and $\mathcal{S}_{2}=$ $\left(E_{k}^{(2)}, A_{k}^{(2)}, B_{k}^{(2)}, C_{k}^{(2)}, D_{k}^{(2)}\right)$ of order $\mathbf{n}_{2}$ we denote with $\mathcal{S}_{1} \star \mathcal{S}_{2}:=\left(\widehat{E}_{k}, \widehat{A}_{k}, \widehat{B}_{k}, \widehat{C}_{k}, \widehat{D}_{k}\right)$ and has a realization of order $\mathbf{n}_{1}+\mathbf{n}_{2}$ with the state-space matrices

$$
\begin{gathered}
\widehat{E}_{k}=\left[\begin{array}{cc}
E_{k}^{(1)} & 0 \\
0 & E_{k}^{(2)}
\end{array}\right], \quad \widehat{A}_{k}=\left[\begin{array}{cc}
A_{k}^{(1)} & B_{k}^{(1)} C_{k}^{(2)} \\
0 & A_{k}^{(2)}
\end{array}\right], \\
\widehat{B}_{k}=\left[\begin{array}{c}
B_{k}^{(1)} D_{k}^{(2)} \\
B_{k}^{(2)}
\end{array}\right], \quad \widehat{C}_{k}=\left[\begin{array}{ll}
C_{k}^{(1)} & D_{k}^{(1)} C_{k}^{(2)}
\end{array}\right], \\
\widehat{D}_{k}=D_{k}^{(1)} D_{k}^{(2)}
\end{gathered}
$$

The parallel coupling of two periodic systems $\mathcal{S}_{1}$ and $\mathcal{S}_{2}$ we denote by $\mathcal{S}_{1} \oplus \mathcal{S}_{2}=\left(\widehat{E}_{k}, \widehat{A}_{k}, \widehat{B}_{k}, \widehat{C}_{k}, \widehat{D}_{k}\right)$ and has a realization of order $\mathbf{n}_{1}+\mathbf{n}_{2}$ with the state-space matrices

$$
\begin{gathered}
\widehat{E}_{k}=\left[\begin{array}{cc}
E_{k}^{(1)} & 0 \\
0 & E_{k}^{(2)}
\end{array}\right], \quad \widehat{A}_{k}=\left[\begin{array}{cc}
A_{k}^{(1)} & 0 \\
0 & A_{k}^{(2)}
\end{array}\right], \\
\widehat{B}_{k}=\left[\begin{array}{c}
B_{k}^{(1)} \\
B_{k}^{(2)}
\end{array}\right], \quad \widehat{C}_{k}=\left[C_{k}^{(1)} C_{k}^{(2)}\right], \quad \widehat{D}_{k}=D_{k}^{(1)}+D_{k}^{(2)}
\end{gathered}
$$


We denote by $\left[\begin{array}{ll}\mathcal{S}_{1} & \mathcal{S}_{2}\end{array}\right]$ and $\left[\begin{array}{l}\mathcal{S}_{1} \\ \mathcal{S}_{2}\end{array}\right]$ the input and output concatenated couplings, respectively, which have obvious state-space representations. For example, $\left[\mathcal{S}_{1} \mathcal{S}_{2}\right]:=$ $\left(\widehat{E}_{k}, \widehat{A}_{k}, \widehat{B}_{k}, \widehat{C}_{k}, \widehat{D}_{k}\right)$ has the realization

$$
\begin{gathered}
\widehat{E}_{k}=\left[\begin{array}{cc}
E_{k}^{(1)} & 0 \\
0 & E_{k}^{(2)}
\end{array}\right], \quad \widehat{A}_{k}=\left[\begin{array}{cc}
A_{k}^{(1)} & 0 \\
0 & A_{k}^{(2)}
\end{array}\right], \\
\widehat{B}_{k}=\left[\begin{array}{cc}
B_{k}^{(1)} & 0 \\
0 & B_{k}^{(2)}
\end{array}\right], \quad \widehat{C}_{k}=\left[\begin{array}{ll}
C_{k}^{(1)} & C_{k}^{(2)}
\end{array}\right], \\
\widehat{D}_{k}=\left[\begin{array}{ll}
D_{k}^{(1)} & D_{k}^{(2)}
\end{array}\right]
\end{gathered}
$$

\section{Inversion and conjugation}

The inverse of $\mathcal{S}=\left(E_{k}, A_{k}, B_{k}, C_{k}, D_{k}\right)$ for invertible $D_{k}$ is

$$
\mathcal{S}^{-1}=\left(E_{k}, A_{k}-B_{k} D_{k}^{-1} C_{k}, D_{k}^{-1} B_{k}, C_{k} D_{k}^{-1}, D_{k}^{-1}\right)
$$

and has order $\mathbf{n}$. More general inverses of periodic systems are discussed in [4].

The conjugate system $\mathcal{S}^{*}$ is defined as $\mathcal{S}^{*}=$ $\left(\widetilde{E}_{k}, \widetilde{A}_{k}, \widetilde{B}_{k}, \widetilde{C}_{k}, \widetilde{D}_{k}\right)$, where (see [5])

$$
\begin{gathered}
\widetilde{E}_{k}=\left[\begin{array}{cc}
A_{k}^{T} & 0 \\
-B_{k}^{T} & 0
\end{array}\right], \quad \widetilde{A}_{k}=\left[\begin{array}{cc}
E_{k-1}^{T} & 0 \\
0 & -I
\end{array}\right], \\
\widetilde{B}_{k}=\left[\begin{array}{c}
-C_{k}^{T} \\
D_{k}^{T}
\end{array}\right], \quad, \widetilde{C}_{k}=\left[\begin{array}{ll}
0 & I
\end{array}\right], \quad \widetilde{D}_{k}=0
\end{gathered}
$$

If the periodic pair $\left(E_{k}, A_{k}\right)$ has constant dimension and $A_{k}$ is invertible, then an equivalent causal descriptor realization of $\mathcal{S}^{*}$ is given by

$$
\mathcal{S}^{*}=\left(A_{k}^{T}, E_{k-1}^{T},-C_{k}^{T}, B_{k}^{T} A_{k}^{-T} E_{k-1}^{T}, D_{k}^{T}-B_{k}^{T} A_{k}^{-T} C_{k}^{T}\right)
$$

\section{E. Lifted representation}

We can formulate the factorization problems addressed in this paper and interpret the obtained results in terms of the transfer-function matrix (TFM) corresponding to the associated stacked lifted representation of [6], which uses the input-state-output behavior of the system over time intervals of length $N$, rather then 1 . The lifted input, output and state vectors are defined as

$$
\begin{aligned}
& \bar{u}_{k}(h)=\left[u^{T}(h N+k) \cdots u^{T}(h N+k+N-1)\right]^{T}, \\
& \bar{y}_{k}(h)=\left[y^{T}(h N+k) \cdots y^{T}(h N+k+N-1)\right]^{T}, \\
& \bar{x}_{k}(h)=\left[x^{T}(h N+k) \cdots x^{T}(h N+k+N-1)\right]^{T}
\end{aligned}
$$

and the corresponding lifted system can be represented by a time-invariant discrete-time descriptor system of the form

$$
\begin{aligned}
\bar{E}_{k} \bar{x}_{k}(h+1) & =\bar{A}_{k} \bar{x}_{k}(h)+\bar{B}_{k} \bar{u}_{k}(h) \\
\bar{y}_{k}(h) & =\bar{C}_{k} \bar{x}_{k}(h)+\bar{D}_{k} \bar{u}_{k}(h)
\end{aligned}
$$

where

$$
\bar{A}_{k}-z \bar{E}_{k}=\left[\begin{array}{ccccc}
A_{k} & -E_{k} & O & \cdots & O \\
O & \ddots & \ddots & \ddots & \vdots \\
\vdots & \ddots & \ddots & -E_{k+N-3} & O \\
O & & \ddots & A_{k+N-2} & -E_{k+N-2} \\
-z E_{k+N-1} & O & \cdots & O & A_{k+N-1}
\end{array}\right]
$$

is the pole pencil of the periodic pair $\left(A_{k}, E_{k}\right)$ and

$$
\begin{aligned}
& \bar{B}_{k}=\operatorname{diag}\left\{B_{k}, \ldots, B_{k+N-1}\right\}, \\
& \bar{C}_{k}=\operatorname{diag}\left\{C_{k}, \ldots, C_{k+N-1}\right\}, \\
& \bar{D}_{k}=\operatorname{diag}\left\{D_{k}, \ldots, D_{k+N-1}\right\} .
\end{aligned}
$$

The $p N \times m N$ TFM of the lifted-system corresponding to $\mathcal{S}$ we denote by

$$
G_{k}^{\mathcal{S}}(z)=\bar{C}_{k}\left(z \bar{E}_{k}-\bar{A}_{k}\right)^{-1} \bar{B}_{k}+\bar{D}_{k}
$$

It can be shown that the TFM $G_{k}^{\mathcal{S}}(z)$ of a causal system $\left(E_{k}\right.$ invertible) is proper and belongs to a class of special type of proper TFMs for which $G_{k}^{\mathcal{S}}(\infty)$ is lower block triangular, with all diagonal blocks of the form $p \times m$. Only TFMs with this property correspond to causal periodic systems.

The previously defined operations with periodic systems: series coupling, parallel coupling, inversion (2), or conjugation, can easily be expressed in terms of TFMs as follows:

$$
\begin{gathered}
G_{k}^{\mathcal{S}_{1} \star \mathcal{S}_{2}}(z)=G_{k}^{\mathcal{S}_{1}}(z) G_{k}^{\mathcal{S}_{2}}(z), \\
G_{k}^{\mathcal{S}_{1} \oplus \mathcal{S}_{2}}(z)=G_{k}^{\mathcal{S}_{1}}(z)+G_{k}^{\mathcal{S}_{2}}(z), \\
G_{k}^{\mathcal{S}^{-1}}(z)=\left(G_{k}^{\mathcal{S}}(z)\right)^{-1}, \\
G_{k}^{\mathcal{S}^{*}}(z)=\left(G_{k}^{\mathcal{S}}(1 / z)\right)^{T} .
\end{gathered}
$$

Important remark. In what follows we tacitly assume that the lifting of input and output concatenated systems is done by preserving the original separation of the inputs and outputs (instead of lifting jointly the inputs and outputs). This has the important consequence that the lifted TFM of an input concatenated system $\left[\mathcal{S}_{1} \mathcal{S}_{2}\right]$ can be built by concatenating the individual lifted TFMs column-wise, i.e.

$$
G_{k}^{\left[\mathcal{S}_{1} \mathcal{S}_{2}\right]}(z)=\left[\begin{array}{ll}
G_{k}^{\mathcal{S}_{1}}(z) & G_{k}^{\mathcal{S}_{2}}(z)
\end{array}\right]
$$

Similarly, the lifted TFM of output concatenated systems can be built by stacking the individual lifted TFMs row-wise.

\section{FACTORIZED REPRESENTATIONS}

In this paper we consider the computation of a right factorized representation of the periodic system $\mathcal{S}$ of the form

$$
\mathcal{S}=\mathcal{N} \star \mathcal{M}^{-1}
$$

or of a left factorized representation of $\mathcal{S}$ of the form

$$
\mathcal{S}=\mathcal{M}^{-1} \star \mathcal{N}
$$

where $\mathcal{M}$ and $\mathcal{N}$ are periodic systems with certain desirable properties (e.g., causality, stability). The lifted TFMs corresponding to the factorized representations (5) and (6) can be expressed similarly as

$$
G_{k}^{\mathcal{S}}(z)=G_{k}^{\mathcal{N}}(z)\left(G_{k}^{\mathcal{M}}(z)\right)^{-1}
$$

or

$$
G_{k}^{\mathcal{S}}(z)=\left(G_{k}^{\mathcal{M}}(z)\right)^{-1} G_{k}^{\mathcal{N}}(z)
$$

respectively.

A factorized representation $\mathcal{S}=\mathcal{N} \star \mathcal{M}^{-1}$ with $\mathcal{N}$ and $\mathcal{M}$ stable, is called a periodic right coprime factorization (PRCF) if there exist stable special proper TFMs $U_{k}(z)$ 
and $V_{k}(z)$ such that $U_{k}(z) G_{k}^{\mathcal{N}}(z)+V_{k}(z) G_{k}^{\mathcal{M}}(z)=I_{m N}$. Analogously, a factorized representation of $\mathcal{S}$ in the form $\mathcal{S}=\mathcal{M}^{-1} \star \mathcal{N}$ with $\mathcal{N}$ and $\mathcal{M}$ stable, is called a periodic left coprime factorization (PLCF) if there exist stable special proper TFMs $U_{k}(z)$ and $V_{k}(z)$ such that $G_{k}^{\mathcal{N}}(z) U_{k}(z)+$ $G_{k}^{\mathcal{M}}(z) V_{k}(z)=I_{p N}$. Stable coprime factorizations have many applications. For example, they are employed in parameterizing the solutions of various synthesis problems, in model reduction of unstable systems [7], or in shaping the dynamics of observers or fault detection filters [8].

A particular case of periodic coprime factorizations is when $\mathcal{M}$ is inner, i.e., $\mathcal{M}^{*} \star \mathcal{M}=\mathcal{I}_{m}$, where $\mathcal{I}_{m}$ is the constant periodic system with $D_{k}=I_{m}$. In this case, $G_{k}^{\mathcal{M}}(z)$, the lifted TFM of the "denominator" factor $\mathcal{M}$, is an inner TFM, i.e., $\left(G_{k}^{\mathcal{M}}(1 / z)\right)^{T} G_{k}^{\mathcal{M}}(z)=I_{m N}$ [9]. Factorizations with "inner denominators" are useful in computing spectral factorizations of periodic systems.

In this paper we propose several numerically reliable state space algorithms to compute PRCFs of the form $\mathcal{S}=$ $\mathcal{N} \star \mathcal{M}^{-1}$, where the factors $\mathcal{N}$ and $\mathcal{M}$ are stable and causal periodic systems. For causal periodic systems, an algorithm based on a recursive generalized Schur technique for eigenvalue assignment of periodic systems is proposed to compute stable PRCF with least order stable $\mathcal{M}$. A similar algorithm is proposed to compute PRCFs with inner denominators. An interesting aspect of the proposed algorithms is their ability to determine factorizations with "denominator" factors $\mathcal{M}$ of minimal order. For non-causal systems, we propose a computational approach for the computation of PRCFs with causal factors which involves as a first step the computation of a maximal right annihilator of an extended system followed by the application of the proposed PRCF techniques for standard periodic systems.

The new procedures are generally applicable regardless the original periodic state space representation of $\mathcal{S}$ is stabilizable/detectable or not. The same algorithms can be also used to compute PLCFs by applying them to the dual system defined by the quintuple

$$
\left(\widetilde{E}_{k}^{T}, A_{N+1-k}^{T}, C_{N+1-k}^{T}, B_{N+1-k}^{T}, D_{N+1-k}^{T}\right),
$$

where $\widetilde{E}_{N}=E_{N}^{T}$ and $\widetilde{E}_{k}=E_{N-k}^{T}$, for $k=1, \ldots, N-1$.

\section{Computation OF PRCF}

The factorization algorithms proposed in this paper rely on simple facts concerning factorized representations, which can be easily established extending standard results for the factorization of descriptor systems (see [10]) to the stacked lifted representations.

Fact 1. Any causal periodic system $\mathcal{S}$ with a stabilizable state-space realization $\left(E_{k}, A_{k}, B_{k}, C_{k}, D_{k}\right)$ has a PRCF given by the following choice of the factors

$$
\begin{aligned}
& \mathcal{N}=\left(E_{k}, A_{k}+B_{k} F_{k}, B_{k} W_{k}, C_{k}+D_{k} F_{k}, D_{k} W_{k}\right) \\
& \mathcal{M}=\left(E_{k}, A_{k}+B_{k} F_{k}, B_{k} W_{k}, F_{k}, W_{k}\right)
\end{aligned}
$$

where $F_{k}$ is chosen such that all characteristic multipliers of the periodic pair $\left(E_{k}, A_{k}+B_{k} F_{k}\right)$ are stable and $W_{k}$ is an arbitrary invertible periodic matrix.
Particular factorizations with inner "denominator" can be determined by suitably choosing $F_{k}$ and $W_{k}$.

The algorithms proposed in this paper use implicitly the more general expressions for the matrices of the factors using the following replacements corresponding to an additional periodic similarity transformation

$$
\begin{aligned}
E_{k} & \leftarrow U_{k} E_{k} V_{k+1}, \\
A_{k}+B_{k} F_{k} & \leftarrow U_{k}\left(A_{k}+B_{k} F_{k}\right) V_{k}, \\
B_{k} W_{k} & \leftarrow U_{k} B_{k} W_{k}, \\
C_{k}+D_{k} F_{k} & \leftarrow\left(C_{k}+D_{k} F_{k}\right) V_{k}, \\
F_{k} & \leftarrow F_{k} V_{k},
\end{aligned}
$$

where $U_{k}$ and $V_{k}$ are periodic orthogonal transformation matrices (usually not explicitly computed) used to obtain the resulting matrices in particular condensed forms.

Fact 2. If $\mathcal{S}=\mathcal{N}_{1} \star \mathcal{M}_{1}^{-1}$ and $\mathcal{N}_{1}=\mathcal{N}_{2} \star \mathcal{M}_{2}^{-1}$, then $\mathcal{S}$ has the fractional representation $\mathcal{S}=\mathcal{N} \star \mathcal{M}^{-1}$, where $\mathcal{N}=\mathcal{N}_{2}$ and $\mathcal{M}=\mathcal{M}_{1} \star \mathcal{M}_{2}$.

This simple fact allows us to obtain explicit formulas to update partial factorizations by using simple state space formulas. Let $\mathcal{N}_{1}$ and $\mathcal{M}_{1}$ be the factors computed as

$$
\begin{aligned}
& \mathcal{N}_{1}=\left(E_{k}, A_{k}+B_{k} F_{k, 1}, B_{k} W_{k, 1}, C_{k}+D_{k} F_{k, 1}, D_{k} W_{k, 1}\right) \\
& \mathcal{M}_{1}=\left(E_{k}, A_{k}+B_{k} F_{k, 1}, B_{k} W_{k, 1}, F_{k, 1}, W_{k, 1}\right)
\end{aligned}
$$

and let $\mathcal{N}_{2}$ and $\mathcal{M}_{2}$ be the factors of $\mathcal{N}_{1}$ computed as

$$
\begin{aligned}
& \mathcal{N}_{2}=\left(E_{k}, A_{k}+B_{k} F_{k}, B_{k} W_{k}, C_{k}+D_{k} F_{k}, D_{k} W_{k}\right) \\
& \mathcal{M}_{2}=\left(E_{k}, A_{k}+B_{k} F_{k}, B_{k} W_{k}, F_{k, 2}, W_{k, 2}\right)
\end{aligned}
$$

where

$$
\begin{aligned}
& F_{k}=F_{k, 1}+W_{k, 1} F_{k, 2} \\
& W_{k}=W_{k, 1} W_{k, 2}
\end{aligned}
$$

It easy to verify that the system $\mathcal{M}_{1} \star \mathcal{M}_{2}$ is given by

$$
\mathcal{M}_{1} \star \mathcal{M}_{2}=\left(E_{k}, A_{k}+B_{k} F_{k}, B_{k} W_{k}, F_{k}, W_{k}\right)
$$

and thus equations (7) serve as explicit updating formulas of fractional representations. These formulas can be extended in a straightforward way to include arbitrary similarity transformation matrices. If $W_{k, 1}=I_{m}$ and $W_{k, 2}=I_{m}$, then the updating formulas reduce to a very simple form

$$
F_{k}=F_{k, 1}+F_{k, 2} .
$$

In this section we propose an algorithm to compute a PRCF $\mathcal{S}=\mathcal{N} \star \mathcal{M}^{-1}$ with a least order $\mathcal{M}$. This algorithm can handle even the case when the original periodic descriptor system representation is not stabilizable. The basis for our algorithm is an eigenvalue assignment procedure for periodic systems described in [11], which on turn relies on similar techniques developed for standard systems [12], [13]. This algorithm has the ability to keep unaltered the stable characteristic multipliers of the periodic pair $\left(E_{k}, A_{k}\right)$ and to move only the unstable ones to stable locations by choosing an appropriate periodic feedback matrix $F_{k}$. An additional useful feature of this algorithm is that simultaneously with the stabilizing $F_{k}$, it determines the generalized periodic real Schur form (GPRSF) of the pair $\left(E_{k}, A_{k}+B_{k} F_{k}\right)$. This makes possible to extract easily a minimal realization for the "denominator" factor $\mathcal{M}$. 
Consider the disjoint partition of the complex plan $\mathbb{C}=$ $\mathbb{C}_{g} \cup \mathbb{C}_{b}$, where $\mathbb{C}_{g}$ and $\mathbb{C}_{b}$ denote the good and bad regions for the characteristic multipliers, respectively. We assume in what follows that $\mathbb{C}_{g}$ contains 0 . The basis of the proposed algorithm is the following extension to time-varying dimensions of the ordered periodic Schur decomposition for periodic matrix pairs [14], [15]:

Theorem 1: Given the $N$-periodic matrices $E_{k} \in$ $\mathbb{R}^{n_{k+1} \times n_{k+1}}, A_{k} \in \mathbb{R}^{n_{k+1} \times n_{k}}$, there exist $N$-periodic orthogonal matrices $Q_{k}$ and $Z_{k}$ such that

$$
\begin{aligned}
\widetilde{A}_{k}:=Q_{k} A_{k} Z_{k} & =\left[\begin{array}{cc}
A_{k}^{g} & A_{k}^{g b} \\
0 & A_{k}^{b}
\end{array}\right], \\
\widetilde{E}_{k}:=Q_{k} E_{k} Z_{k+1} & =\left[\begin{array}{cc}
E_{k}^{g} & E_{k}^{g b} \\
0 & E_{k}^{b}
\end{array}\right],
\end{aligned}
$$

where $\left(A_{k}^{g}, E_{k}^{g}\right)$ contains the characteristic multipliers in the good region $\mathbb{C}_{g}$ and $\left(A_{k}^{b}, E_{k}^{b}\right)$ contains the characteristic multipliers in the bad region $\mathbb{C}_{b}$, with $E_{k}^{b} \in \mathbb{R}^{n_{b} \times n_{b}}, A_{k}^{b} \in$ $\mathbb{R}^{n_{b} \times n_{b}}$. The periodic pair $\left(\widetilde{E}_{k}, \widetilde{A}_{k}\right)$ is in a GPRSF, with $\widetilde{E}_{k}$ upper triangular and $\widetilde{A}_{k}$ in a periodic quasi-triangular (real Schur) form.

This decomposition ensures that the trailing periodic pair $\left(A_{k}^{b}, E_{k}^{b}\right)$ has constant dimensions, although the original pair may have time-varying dimensions. The reason for that is that all zero characteristic multipliers belong to the leading periodic pair $\left(A_{k}^{g}, E_{k}^{g}\right)$, which has in general time-varying dimensions.

The computation of the ordered GPRSF (9) and (10) can be performed by reducing first the pair $\left(E_{k}, A_{k}\right)$ to a separated form similar to (9) and (10), where the core characteristic multipliers appear in the trailing $\underline{n} \times \underline{n}$ part and the leading part contains the structurally null characteristic multipliers. The trailing pair (with constant dimensions) is then reduced to a GPRSF using the algorithms proposed in [14], [15], while eigenvalue reordering techniques for matrix products are employed to achieve the desired spectral separation [16].

The following algorithm can be used to compute a PRCF of a periodic system $\mathcal{S}$.

\section{PRCF Algorithm.}

1. Find orthogonal $N$-periodic matrices $Q_{k}$ and $Z_{k}$ to reduce the pair $\left(E_{k}, A_{k}\right)$ to the ordered GPRSF $\left(\widetilde{E}_{k}, \widetilde{A}_{k}\right)$ in (9) and (10). For $k=1, \ldots, N$, compute $\widetilde{B}_{k}=Q_{k} B_{k}, \widetilde{C}_{k}=$ $C_{k} Z_{k}$ and set $\widetilde{F}_{k}=0, \widetilde{W}_{k}=I_{m}$.

2. If $n_{b}=0$, go to 7 .

3. Let $\left(\eta_{k}, \alpha_{k}\right)$ be the last diagonal blocks of $\left(\widetilde{E}_{k}, \widetilde{A}_{k}\right)$ of order $\ell=1$ or $\ell=2$ and let $\beta_{k}$ be the $\ell \times m$ matrix formed from the last $\ell$ rows of $\widetilde{B}_{k}$. If for $k=1, \ldots, N,\left\|\beta_{k}\right\| \leq \epsilon$ (a given tolerance), then $n_{k} \leftarrow n_{k}-\ell, n_{b} \leftarrow n_{b}-\ell$ and go to 2 .

4. Choose an appropriate $\ell \times \ell N$-periodic matrix $\gamma_{k}$ such that $\Lambda\left(\Psi_{\gamma_{k}}\right) \subset \mathbb{C}_{g}$; for $k=1, \ldots, N$, compute $\kappa_{k}$ such that $\beta_{k} \kappa_{k}=\eta_{k} \gamma_{k}-\alpha_{k}$ and set $K_{k}=\left[\begin{array}{ll}0 & \kappa_{k}\end{array}\right], V_{k}=I_{m}$.

5. For $k=1, \ldots, N$, update matrices: $\widetilde{A}_{k} \leftarrow \widetilde{A}_{k}+\widetilde{B}_{k} K_{k}$, $\widetilde{F}_{k} \leftarrow \widetilde{F}_{k}+\widetilde{W}_{k} K_{k}, W_{k} \leftarrow \widetilde{W}_{k} V_{k}$.
6. Compute the orthogonal matrices $\widetilde{Q}_{k}$ and $\widetilde{Z}_{k}$ to move the last $\ell \times \ell$ diagonal blocks of $\left(\widetilde{E}_{k}, \widetilde{A}_{k}\right)$ to row positions $n_{k+1}-n_{b}+1$ by interchanging the diagonal blocks of the GPRSF. Compute $\widetilde{E}_{k} \leftarrow \widetilde{Q}_{\underline{\varepsilon}} \widetilde{E}_{k} \widetilde{Z}_{k+1}, \widetilde{A}_{k} \leftarrow \widetilde{Q}_{k} \widetilde{A}_{k} \widetilde{Z}_{k}$, $\widetilde{B}_{k} \leftarrow \widetilde{Q}_{k} \widetilde{B}_{k}, \widetilde{C}_{k} \leftarrow \widetilde{C}_{k} \widetilde{Z}_{k}, \widetilde{F}_{k} \leftarrow \widetilde{F}_{k} \widetilde{Z}_{k}$. Put $n_{b} \leftarrow n_{b}-\ell$ and go to 2 .

$$
\text { 7. Put } \begin{aligned}
\mathcal{N} & =\left(\widetilde{E}_{k}, \widetilde{A}_{k}, \widetilde{B}_{k} \widetilde{W}_{k}, \widetilde{C}_{k}+D_{k} \widetilde{F}_{k}, D_{k} \widetilde{W}_{k}\right), \\
\mathcal{M} & =\left(\widetilde{E}_{k}, \widetilde{A}_{k}, \widetilde{B}_{k} \widetilde{W}_{k}, \widetilde{F}_{k}, \widetilde{W}_{k}\right) .
\end{aligned}
$$

This algorithm can be viewed as a recursive updating procedure of an initial fractional representation $\mathcal{S}=\mathcal{N}_{0} \star \mathcal{M}_{0}^{-1}$ with $\mathcal{N}_{0}=\mathcal{S}$ and $\mathcal{M}_{0}=I_{m}$, by using the simple updating formula (8) combined with orthogonal coordinate transformations performed on the matrices of partial factorizations. The matrix pairs $\left(\widetilde{E}_{k}, \widetilde{A}_{k}\right)$ in the initial factorization of $\mathcal{S}$ is in a GPRSF (computed at step 1) and this form is preserved at subsequent steps. The resulting final pair $\left(\widetilde{E}_{k}, \widetilde{A}_{k}\right)$ is therefore in a GPRSF and if the original system is stabilizable, then $\widetilde{E}_{k}$ and $\widetilde{A}_{k}$ contain the matrices $U_{k} E_{k} V_{k+1}$ and $U_{k}\left(A_{k}+B_{k} F_{k}\right) V_{k}$, respectively, where $U_{k}$ and $V_{k}$ are the accumulated orthogonal transformations performed at steps 1 and 6 of the algorithm, and $F_{k}$ is the periodic stabilizing state feedback matrix $\widetilde{F}_{k} V_{k}^{T}$. If the original system is not stabilizable, then the unstabilizable blocks are detected at step 3 and the corresponding unstabilizable parts are deflated by simply decreasing the order of the system with $\ell$. If unstabilizable blocks are detected by the algorithm then the resulting factors have order less than $\mathbf{n}$.

The resulting form of matrices of the computed factors allows to easily determine a minimal realization of $\mathcal{M}$. The resulting $\widetilde{F}_{k}$ has always the form

$$
\widetilde{F}_{k}=\left[0 \widetilde{F}_{k, 2}\right],
$$

where the number of columns of $\widetilde{F}_{k, 2}$ is equal to the number of unstable reachable characteristic multipliers of the pair $\left(E_{k}, A_{k}\right)$. If we partition conformably $\widetilde{E}_{k}, \widetilde{A}_{k}$ and $\widetilde{B}_{k}$

$\widetilde{E}_{k}=\left[\begin{array}{cc}\widetilde{E}_{k, 11} & \widetilde{E}_{k, 12} \\ 0 & \widetilde{E}_{k, 22}\end{array}\right], \widetilde{A}_{k}=\left[\begin{array}{cc}\widetilde{A}_{k, 11} & \widetilde{A}_{k, 12} \\ 0 & \widetilde{A}_{k, 22}\end{array}\right], \widetilde{B}=\left[\begin{array}{c}\widetilde{B}_{k, 1} \\ \widetilde{B}_{k, 2}\end{array}\right]$,

then $\left(\widetilde{E}_{k, 22}, \widetilde{A}_{k, 22}, \widetilde{B}_{k, 2} \widetilde{W}_{k}, \widetilde{F}_{k, 2}, \widetilde{W}_{k}\right)$ is the least order realization of $\mathcal{M}$. Notice however that the order of the realization of $\mathcal{M}$ can be higher than the least possible order if some unstable characteristic multipliers of $\left(E_{k}, A_{k}\right)$ are reachable but not observable.

The PRCF Algorithm is based on a generalization of a eigenvalue assignment algorithm for causal periodic systems [11], which extends the pole assignment algorithm of [12]. By extending the roundoff error analysis of that algorithm [17] to the periodic case, we can state similarly that if each partial feedback $K_{k}$ computed at step 4 satisfies $\left\|K_{k}\right\| \leq$ $\left\|A_{k}\right\| /\left\|B_{k}\right\|$, then the eigenvalue assignment algorithm is numerically backward stable. This condition is also applicable in our case, because it is independent of the presence of $E_{k}$. We note however that unfortunately this condition can not be fulfilled if large gains are necessary to stabilize the system. This can arise either if the unstable characteristic multipliers 
are too "far" from the stable region or if some characteristic multipliers are nearly unreachable.

\section{PRCF WITH INNER DENOMINATOR}

We assume in this section that $\mathcal{S}$ has no characteristic multipliers on the unit circle. The algorithm to compute the periodic right coprime factorization with inner denominator (PRCFID) of $\mathcal{S}$ is similar to the PRCF Algorithm, and here we will show only the differences to this algorithm. We will use the following definitions of good and bad stability regions

$$
\mathbb{C}_{g}=\mathbb{D}^{-}, \quad \mathbb{C}_{b}=\mathbb{C} \backslash \mathbb{D}^{-},
$$

where $\mathbb{D}^{-}$is the interior of the unit circle. Furthermore, the updating formulas of Fact 2 rely this time on using low order inner denominator factors $\mathcal{M}_{1}$ and $\mathcal{M}_{2}$, and consequently the resulting factor $\mathcal{M}=\mathcal{M}_{1} \star \mathcal{M}_{2}$ is inner too. Finally, for the computation of these inner factors we will use the following formulas to compute the PRCFID of a particular class of systems [7].

Fact 4. Let $\mathcal{S}=\left(E_{k}, A_{k}, B_{k}, C_{k}, D_{k}\right)$ be a reachable periodic descriptor representation with $E_{k}$ nonsingular and $\Lambda\left(\Psi_{E_{k}^{-1} A_{k}}\right) \in \mathbb{C}_{b}$. Then $\mathcal{M}=\left(E_{k}, A_{k}+\right.$ $\left.B_{k} F_{k}, B_{k} W_{k}, F_{k}, W_{k}\right)$ is inner by choosing $F_{k}$ and $W_{k}$ as:

$$
\begin{aligned}
& F_{k}=-B_{k}^{T}\left(E_{k} Y_{k+1} E_{k}^{T}+B_{k} B_{k}^{T}\right)^{-1} A_{k} \\
& W_{k}=\left(I+B_{k}^{T}\left(E_{k} Y_{k+1} E_{k}^{T}\right)^{-1} B_{k}\right)^{-1 / 2}
\end{aligned}
$$

where, for $k=1, \ldots, N, Y_{k}$ satisfy the periodic generalized Lyapunov equation

$$
A_{k} Y_{k} A_{k}^{T}-B_{k} B_{k}^{T}=E_{k} Y_{k+1} E_{k}^{T}
$$

The above expressions for $F_{k}$ and $W_{k}$ represent straightforward transcriptions of analogous formulas for standard systems [18] and rely on formulas developed in [19] and [7]. Note that only $E_{k}, A_{k}$ and $B_{k}$ are involved in these expressions. The PRCFID Algorithm is identical to the PRCF Algorithm excepting Step 4, where the above formulas are employed to determine the feedback $\kappa_{k}$ and gain $V_{k}$. Step 4 thus becomes:

4. For the system $\left(\eta_{k}, \alpha_{k}, \beta_{k}, *, *\right)$ compute periodic $\kappa_{k}$ and $V_{k}$ such that $\left(\eta_{k}, \alpha_{k}+\beta_{k} \kappa_{k}, \beta_{k} V_{k}, \kappa_{k}, V_{k}\right)$ is inner. For $k=1, \ldots, N$, set $K_{k}=\left[0 \kappa_{k}\right]$.

A minimal realization for the inner factor $\mathcal{M}$ can be determined from the partitioning (11) and (12) of the resulting $\widetilde{F}_{k}, \widetilde{E}_{k}, \widetilde{A}_{k}$ and $\widetilde{B}_{k}$ and is given by $\mathcal{M}=$ $\left(\widetilde{E}_{k, 22}, \widetilde{A}_{k, 22}, \widetilde{B}_{k, 2} \widetilde{W}_{k}, \widetilde{F}_{k, 2}, \widetilde{W}_{k}\right)$.

The PRCFID Algorithm can be viewed as an eigenvalue assignment algorithm which assigns the unstable characteristic multipliers in symmetrical positions with respect to the unit circle. Because practically there is no freedom in assigning the characteristic multipliers, it is to be expected that the algorithm performs in a numerically stable way only if the norms of the elementary feedback matrices $K_{k}$ computed at step 4 are not too high.

Remark. If the system $\mathcal{S}=\left(E_{k}, A_{k}, B_{k}, C_{k}, D_{k}\right)$ is square, with invertible $D_{k}$, then the PRCFID Algorithm can be used to compute an inner-outer factorization $\mathcal{S}=\mathcal{M} \star \mathcal{N}$, where $\mathcal{M}$ is inner and $\mathcal{N}$ is outer (minimum-phase and stable). This can be done similarly as for standard systems [20], by applying the PRCFID Algorithm to the inverse system $\mathcal{S}^{-1}$ in (2).

\section{Computation of CAUSAL PRCF}

If the given periodic system $\mathcal{S}=\left(E_{k}, A_{k}, B_{k}, C_{k}, D_{k}\right)$ is non-causal, that is, the pole pencil (4) has infinite zeros, it is of interest in several applications to compute a PRCF $\mathcal{S}=\mathcal{N} \star \mathcal{M}^{-1}$ such that both factors are causal and stable periodic systems. For this purpose we can use the following conceptually simple approach to compute a causal periodic right coprime factorization (CPRCF):

\section{CPRCF Algorithm.}

1. Compute a factorization $\mathcal{S}=\mathcal{N}_{1} \star \mathcal{M}_{1}^{-1}$, where both factors are causal (but possibly unstable).

2. Compute the PRCF $\left[\begin{array}{l}\mathcal{N}_{1} \\ \mathcal{M}_{1}\end{array}\right]=\left[\begin{array}{c}\mathcal{N} \\ \mathcal{M}\end{array}\right] \star \widetilde{\mathcal{M}}_{2}^{-1}$ by using the PRCF Algorithm.

It is easy to see that $\mathcal{S}=\mathcal{N} \star \mathcal{M}^{-1}$ is the desired CPRCF.

To perform Step 1 we can extend to the periodic case a recently proposed technique to compute proper factorizations of improper systems [21]. We can rewrite $\mathcal{S}=\mathcal{N}_{1} \star \mathcal{M}_{1}^{-1}$ as

$$
\left[\begin{array}{ll}
\mathcal{S} & -\mathcal{I}_{p}
\end{array}\right] \star\left[\begin{array}{c}
\mathcal{M}_{1} \\
\mathcal{N}_{1}
\end{array}\right]=0
$$

Thus, we can determine the compound system

$$
\mathcal{R}:=\left[\begin{array}{c}
\mathcal{M}_{1} \\
\mathcal{N}_{1}
\end{array}\right]
$$

as a maximal right annihilator (i.e., with $p+m$ outputs and $m$ inputs) of the right invertible periodic system $\left[\mathcal{S}-\mathcal{I}_{p}\right]$. Equivalently, the lifted TFM $G^{\mathcal{R}}(z)$ is a right rational nullspace basis of $\left[G^{\mathcal{S}}(z)-I_{p N}\right]$. The determination of $\mathcal{R}$ can be done using the method proposed in [8] to compute least order annihilators for periodic systems. The invertibility of $\mathcal{M}_{1}$, or equivalently of $G^{\mathcal{M}_{1}}(z)$, is guaranteed by Lemma 2 of [22] by observing that the lifted TFM $G^{\mathcal{R}}(z)$ is a right nullspace basis, and thus has full column rank.

The computation of left annihilators for periodic systems has been addressed in [8] in the context of fault detection. Here we only sketch the corresponding procedure to determine a right annihilator of $\left[\mathcal{S}-\mathcal{I}_{p}\right]$ for which we employ the extended periodic pair $\left(S_{k}, T_{k}\right)$ defined as

$$
S_{k}=\left[\begin{array}{ccc}
A_{k} & B_{k} & 0 \\
C_{k} & D_{k} & -I_{p}
\end{array}\right], \quad T_{k}=\left[\begin{array}{ccc}
E_{k} & O & O \\
O & O & O
\end{array}\right]
$$

Let $Q_{k}$ and $Z_{k}$ be orthogonal $N$-periodic matrices determined using the algorithm proposed in [23] to reduce the $N$-periodic pair $\left(S_{k}, T_{k}\right)$ to the Kronecker-like form $\left(\bar{S}_{k}, \bar{T}_{k}\right):=\left(Q_{k} S_{k} Z_{k}, Q_{k} T_{k} Z_{k+1}\right)$, where

$$
\bar{S}_{k}=\left[\begin{array}{c|ccc}
B_{k}^{r} & A_{k}^{r} & * & * \\
O & O & A_{k}^{r e g} & * \\
O & O & O & A_{k}^{l} \\
\hline O & O & O & C_{k}^{l}
\end{array}\right]
$$




$$
\bar{T}_{k}=\left[\begin{array}{c|ccc}
O & E_{k}^{r} & * & * \\
O & O & E_{k}^{r e g} & * \\
O & O & O & E_{k}^{l} \\
\hline O & O & O & O
\end{array}\right]
$$

where: (a) the periodic system $\left(E_{k}^{r}, A_{k}^{r}, B_{k}^{r}, *, *\right)$ is completely reachable and $E_{k}^{r}$ is invertible; (b) the periodic system $\left(E_{k}^{l}, A_{k}^{l}, *, C_{k}^{l}, *\right)$ is completely observable and $E_{k}^{l}$ is invertible; (c) the pole pencil, of the form (4), corresponding to the periodic pair $\left(E_{k}^{r e g}, A_{k}^{r e g}\right)$ is regular. If we partition

$$
\left[\begin{array}{ll}
0 & I_{m+p}
\end{array}\right] Z_{k}=\left[\begin{array}{llll}
D_{k}^{r} & C_{k}^{r} & * & *
\end{array}\right]
$$

in accordance with the column partitioning of $\bar{S}_{k}$ in (14), we obtain that $\mathcal{R}:=\left(A_{k}^{r}, E_{k}^{r}, B_{k}^{r}, C_{k}^{r}, D_{k}^{r}\right)$, with $E_{k}^{r}$ nonsingular, is a periodic descriptor system representation for a maximal right annihilator of $\left[\mathcal{S}-\mathcal{I}_{p}\right]$. If we partition now $C_{k}^{r}$ and $D_{k}^{r}$ as

$$
C_{k}^{r}=\left[\begin{array}{c}
C_{k}^{r, \mathcal{M}} \\
C_{k}^{r, \mathcal{N}}
\end{array}\right], \quad D_{k}^{r}=\left[\begin{array}{c}
D_{k}^{r, \mathcal{M}} \\
D_{k}^{r, \mathcal{N}}
\end{array}\right],
$$

where the leading matrices have $m$ rows, then the periodic realizations of the causal factors are

$$
\begin{aligned}
\mathcal{N}_{1} & =\left(A_{k}^{r}, E_{k}^{r}, B_{k}^{r}, C_{k}^{r, \mathcal{N}}, D_{k}^{r, \mathcal{N}}\right) \\
\mathcal{M}_{1} & =\left(A_{k}^{r}, E_{k}^{r}, B_{k}^{r}, C_{k}^{r, \mathcal{M}}, D_{k}^{r, \mathcal{M}}\right)
\end{aligned}
$$

The characteristic multipliers of the factors can be arbitrarily assigned using an additional pencil transformation (see [23] for details). This is is equivalent to use a periodic state feedback $F_{k}$ (see Fact 1) to obtain

$$
\begin{aligned}
\mathcal{N} & =\left(A_{k}^{r}+B_{k}^{r} F_{k}, E_{k}^{r}, B_{k}^{r}, C_{k}^{r, \mathcal{N}}+D_{k}^{r, \mathcal{N}} F_{k}, D_{k}^{r, \mathcal{N}}\right) \\
\mathcal{M} & =\left(A_{k}^{r}+B_{k}^{r} F_{k}, E_{k}^{r}, B_{k}^{r}, C_{k}^{r, \mathcal{M}}+D_{k}^{r, \mathcal{M}} F_{k}, D_{k}^{r, \mathcal{M}}\right)
\end{aligned}
$$

Alternatively, the PRCF Algorithm can be employed as described at Step 2 of the CPRCF Algorithm.

\section{CONCLUSIONS}

Efficient numerically reliable algorithms for computing PRCFs have been proposed. The algorithms are based on a recursive generalized periodic Schur technique for eigenvalue assignment. For the computation of causal PRCFs an algorithm has been proposed which relies on a new technique to compute left/right annihilators of periodic systems. Using such a factorization as a prelimnary step, the computation of normalized periodic coprime factorization for arbitrary periodic systems (causal or not) can be easily performed by solving additionally a spectral factorization problem (along the approach proposed in [24] for standard systems). All proposed algorithms can be also applied to standard causal periodic systems (with $E_{k}=I_{n_{k+1}}$ ), with obvious simplifications leading to an increased computational efficiency.

\section{REFERENCES}

[1] V. Estruch, V. Hernandez, E. Sanchez, and C. Coll, "Forward/backward periodic realizations of nonproper rational matrices," J. Math. Syst. Estim. Control, vol. 8, pp. 155-175, 1998.

[2] A. Varga and P. Van Dooren, "Computing the zeros of periodic descriptor systems," Systems \& Control Lett., vol. 50, pp. 371-381, 2003.

[3] A. Varga, "Strongly stable algorithm for computing periodic system zeros," Proc. of CDC'2003, Maui, Hawaii, 2003.

[4] - "Computation of generalized inverses of periodic systems," Proc. of CDC'04, Paradise Island, Bahamas, 2004.

[5] — " "Computation of $\mathcal{L}_{\infty}$-norm of linear discrete-time periodic systems," Proc. of MTNS'06, Kyoto, Japan, 2006.

[6] O. M. Grasselli and S. Longhi, "Finite zero structure of linear periodic discrete-time systems," Int. J. Systems Sci., vol. 22, pp. 1785-1806, 1991.

[7] A. Varga, "On balancing and order reduction of unstable periodic systems," Proc. of IFAC Workshop on Periodic Control Systems, Como, Italy, 2001, pp. 177-182.

[8] — , "Design of fault detection filters for periodic systems," Proc. of CDC'04, Paradise Island, Bahamas, 2004.

[9] K. Zhou, J. C. Doyle, and K. Glover, Robust and Optimal Control. Prentice Hall, 1996.

[10] A. Varga, "Computation of coprime factorizations of rational matrices," Lin. Alg. \& Appl., vol. 271, pp. 83-115, 1998.

[11] J. Sreedhar and P. Van Dooren, "Pole placement via the periodic Schur decomposition," Proc. 1993 American Control Conference, San Francisco, CA, 1993, pp. 1563-1567.

[12] A. Varga, "A Schur method for pole assignment," IEEE Trans. Automat. Control, vol. 26, pp. 517-519, 1981.

[13] — "On stabilization of descriptor systems," Systems \& Control Letters, vol. 24, pp. 133-138, 1995.

[14] A. W. Bojanczyk, G. Golub, and P. Van Dooren, "The periodic Schur decomposition. Algorithms and applications," Proceedings SPIE Conference, San Diego, F. T. Luk, Ed., vol. 1770, July 1992, pp. 3142.

[15] J. J. Hench and A. J. Laub, "Numerical solution of the discrete-time periodic Riccati equation," IEEE Trans. Automat. Control, vol. 39, pp. 1197-1210, 1994.

[16] R. Granat, B. Kågström, and D. Kressner, "Computing periodic deflating subspaces associated with a specified set of eigenvalues," BIT, vol. 47, pp. 763-791, 2007.

[17] A. Varga, "The numerical stability of an algorithm for pole assignment," Proc. 2-nd IFAC CADCS Symp., West Lafayette, Indiana, G. Leininger (Ed.) Pergamon Press, Oxford, 1982, pp. 117-122.

[18] - "A Schur method for computing coprime factorizations with inner denominators and applications in model reduction," Proc. 1993 American Control Conference, San Francisco, CA, 1993, pp. 21302131.

[19] B. Xie, R. K. A. V. Aripirala, and V. Syrmos, "Model reduction of linear discrete-time periodic systems using hankel-norm approximations," Proc. 13th IFAC Congress, San Francisco, USA, 1996, pp. 245-250.

[20] A. Varga, "Computation of inner-outer factorizations of rational matrices," IEEE Trans. Automat. Control, vol. 43, pp. 684-688, 1998.

[21] _ "On computing minimal proper nullspace bases with applications in fault detection," in Numerical Linear Algebra in Signals, Systems and Control, Ser. Lecture Notes in Electrical Engineering, S. Bhattacharyya, R. Chan, V. Olshewsky, A. Routray, and P. V. Dooren (Eds.) Springer Verlag, Berlin, 2009, (to appear).

[22] G. Verghese, P. Van Dooren, and T. Kailath, "Properties of the system matrix of a generalized state-space system," Int. J. Control, vol. 30, pp. 235-243, 1979.

[23] A. Varga, "Computation of Kronecker-like forms of periodic matrix pairs," Proc. of MTNS'04, Leuven, Belgium, 2004.

[24] $\_$, "Computation of normalized coprime factorizations of rational matrices," Systems \& Control Lett., vol. 33, pp. 37-45, 1998. 